\title{
Caracterización clínica de adultos menores y mayores de 50 años hospitalizados por influenza A H1N1 2009 en un centro hospitalario privado en Santiago, Chile
}

\author{
Franz Baehr M., Gissella Morín R., José A. Del Solar H., Henry Olivi R. y Juan P. Torres T.
}

\section{Clinical features of adults younger and older than 50 years hospitalized with influenza A H1N1 2009 in a private hospital in Santiago, Chile}

Introduction: The new pandemic influenza A H1N1 2009 virus has shown a different clinical behavior in different age groups. Objective: To describe the clinical features of adult patients hospitalized with influenza A H1N1 2009 and compare the clinical and demographic variables among adults $<50$ years and over 50 years old. Patients and Method: We included hospitalized patients between May 17 and July 17, 2009 confirmed by polymerase chain reaction (PCR) and/or rapid test for influenza A. Results: 124 patients were hospitalized with a median age of 33 years (range: 15-82 years). 36\% had comorbidities and the main symptoms were fever, cough, myalgia and sore throat. There were 16 cases with pneumonia. From 124 patients, $77 \%$ were A-49 and 23\% A-50, with comorbidity of $21 \%$ and $86 \%$, respectively. There were no fatalities. Conclusions: Hospitalized patients had mild to moderate disease, a benign course and short hospitalization stay. There were more hospitalizations in A-49 and higher comorbidity in A-50.

Key words: Influenza A H1N1 2009, adults, hospitalized

Palabras clave: virus influenza A H1N1 2009, adultos, hospitalizados.

\section{Introducción}

$\mathrm{E}$ n abril del 2009 se comunicaron los primeros casos de la nueva influenza A H1N1 2009 en el hemisferio norte, que rápidamente adquirió caracteres de pandemia ${ }^{1}$. En nuestro país, el primer caso fue reportado el 17 de mayo y el informe sobre la situación de la infección al 17 de julio describía 10.926 casos confirmados a nivel nacional, de los cuales, 3.086 correspondían a casos confirmados en el Instituto de Salud Pública y 7.840 eran casos confirmados en establecimientos privados.

Se trata de una variante antigénica de influenza A H1N1 que no había sido previamente detectada en cerdos o humanos. Su genoma contiene un segmento humano, un segmento aviar, un segmento porcino americano y otro segmento porcino eurasiático ${ }^{2}$.

El virus afectó en mayor número a personas jóvenes entre 6 y 18 años, al contrario de lo comunicado en la influenza estacional de los últimos años ${ }^{2}$.

Estudios preliminares indican que no hay diferencia en la virulencia de virus obtenidos de pulmones de pacientes con compromiso respiratorio grave de los obtenidos en pacientes con cuadros leves, lo que sugiere que son las personas en sus diferentes etapas de la vida las que reaccionan en forma diferente frente al mismo virus ${ }^{3}$.
Se han observado diferentes grados de severidad, desde casos asintomáticos hasta casos graves, con neumonía y síndrome de distress respiratorio agudo (SDRA). Se describe que en 20 a $25 \%$ de los casos puede existir compromiso digestivo, algo poco habitual en la influenza estacional ${ }^{2,4}$.

Con la llegada del virus pandémico al hemisferio sur en la época invernal se desconocía cuál sería su comportamiento y qué sucedería al presentarse junto con otros virus respiratorios, como el virus respiratorio sincicial y los otros virus de la influenza estacional ${ }^{5}$. Se desconocía también cómo se afectaría el grupo de personas mayores al presentar la infección por la nueva influenza A H1N1 2009.

Este estudio describe las características demográficas y clínicas de los pacientes adultos que se hospitalizaron por influenza A H1N1 2009 en un centro hospitalario privado (Clínica Las Condes, CLC) y la comparación de la infección entre menores de 50 años y mayores de 50 años.

\section{Pacientes y Método}

Clínica Las Condes es el segundo hospital privado más grande en Santiago, ubicado en el sector oriente de
Clínica Las Condes, Santiago, Chile.

Centro de Enfermedades Respiratorias (FBM, GMR, JADS, HOR),

Sub Dirección de Investigación (JPTT)

Recibido: 15 de diciembre de 2009 Aceptado: 30 de marzo de 2010

Correspondencia a: Franz Baehr M. fbaehr@clc.cl 
la ciudad. Cuenta con 204 camas para adultos y 51 camas para niños. Tiene la mayor experiencia en el tratamiento de pacientes con ECMO (Extracorporeal Membrane Oxygenation) en Chile ${ }^{8}$.

Se realizó un estudio descriptivo de una serie de casos en CLC, en pacientes con influenza A H1N1 2009 que fueron hospitalizados entre el 17 de mayo y el 17 de julio del 2009. Para la hospitalización de pacientes se siguió la Guía Clínica Nacional del Ministerio de Salud ${ }^{9}$, aunque en algunos casos se debió a una razón epidemiológica con fines de aislamiento, como sucedió en los primeros casos.

Para este estudio se incluyeron aquellos pacientes adultos, sobre 15 años de edad, hospitalizados y con diagnóstico de influenza A H1N1 2009 confirmado mediante prueba rápida (inmunocromatografía) y/o RPC positiva para influenza A H1N1 2009.

Se excluyeron aquellos pacientes con cuadro clínico de influenza que no fueron confirmados por las técnicas antes descritas o quienes fueron confirmados fuera del período de observación.

El estudio fue aprobado por el Comité de Ética de CLC.

Definición de caso confirmado de influenza A H1N1 2009. Correspondió a aquellos pacientes con síntomas de tipo influenza y con prueba rápida para influenza $\mathrm{A}$ y/o RPC positiva para influenza A H1N1 2009 en una muestra de aspirado nasofaríngeo. La prueba rápida para influenza A se consideró diagnóstica de influenza A H1N1 2009 dado que nuestro laboratorio documentó que hubo una correlación de 97,6\% en 196 muestras positivas por la prueba rápida con la RPC para influenza A H1N1 2009.

Recolección de muestras y técnicas de laboratorio utilizadas para confirmar los casos. Se obtuvieron muestras de aspirado nasofaríngeo para realizar todas las técnicas. La prueba de detección rápida para antígenos de influenza A y B (Quickvue, Quidel, USA) se realizó de acuerdo a las instrucciones del fabricante ${ }^{10}$. La RPC para la detección del virus influenza A H1N1 2009 (Real time ready RT-PCR Influenza A H1N1 detection set ${ }^{\mathrm{TM}}$, Roche, Germany) se basó en la amplificación en tiempo real del gen de la proteína de matriz 2 y la identificación de subtipo en la amplificación del gen de la hemaglutinina HA 1. Esta técnica se realizó de acuerdo a las instrucciones del fabricante, con una correlación de $100 \%$ de las muestras positivas en CLC con el Instituto de Salud Pública de Chile (ISP). En un subgrupo de pacientes se realizó complementariamente estudio con inmunofluorescencia directa para la detección de influenza A y B, virus respiratorio sincicial, adenovirus y parainfluenza 1 , 2 y 3 (Millipore (C), Light Diagnostics, UK). Si la prueba rápida resultó negativa, se realizó estudio con RPC de la misma muestra de secreción obtenida a través de aspirado nasofaríngeo, dentro de las 24 horas de haberla obtenido.
En todos los pacientes se analizó: género, edad, signos y síntomas clínicos principales, antecedentes de vacunación para influenza estacional en el año 2009, motivo de hospitalización, co-morbilidad, fecha de inicio de síntomas, latencia en la consulta (definida como el tiempo transcurrido desde el inicio de los síntomas hasta la fecha de consulta/hospitalización) y tiempo de hospitalización.

Se compararon estas variables entre el grupo $<50$ y el grupo $\geq 50$.

La información se obtuvo de la revisión de fichas clínicas, formularios de notificación inmediata para vigilancia de influenza A H1N1 2009 del Ministerio de Salud (MINSAL) y base de datos de CLC.

Análisis de los datos. La comparación de las variables entre grupos se realizó por prueba de T-Student o prueba de Mann-Whitney, de acuerdo a la distribución de los datos. Se utilizó Chi-cuadrado con corrección de Yates o prueba exacta de Fisher para la asociación entre variables categóricas. La significancia estadística utilizada fue de $p<0,05$. Para todos los análisis estadísticos se utilizó el programa Sigma Stat 3.0 (SPSS Science, Chicago, IL).

\section{Resultados}

Entre el 17 de mayo y el 17 de julio del 2009, hubo 27.308 consultas en Urgencia de CLC por cuadros respiratorios, de las cuales 10.048 tuvieron diagnóstico clínico de influenza. Se confirmaron mediante los métodos ya descritos 4.404 casos de influenza A H1N1 2009. Se hospitalizaron 199 pacientes $(4,5 \%)$, de los cuales 124 $(62,3 \%)$ eran adultos. De estos últimos, 95 correspondieron $\mathrm{a}<50$ y $29 \mathrm{a} \geq 50$ (Figura 1). Todos los pacientes fueron hospitalizados en la primera consulta.

Del total de pacientes hubo predominio femenino (64\%), una mediana de edad de 33 años (rango: 15-82 años). La mediana de latencia fue de 2 días (p25-75\%= 1-4). El principal motivo de hospitalización se debió a compromiso del estado general (CEG) secundario a influenza (65\%), seguido de afecciones respiratorias (21\%). Un 9\% tuvo compromiso digestivo, 2\% manifestaciones neurológicas y $1 \%$ manifestaciones cardiológicas, hematológicas y otorrinolaringológicas, respectivamente. Destaca el diagnóstico de 16 casos de neumonías, basado en la presencia de infiltrados radiológicos más fiebre y/o tos y/o leucocitosis. En la mitad de estos casos se observó clínica de obstrucción bronquial (Tabla 1).

La mediana de días de hospitalización fue de 2 días (p25-75\% =2-3). Los signos y síntomas clínicos más frecuentes fueron fiebre $(75 \%)$, tos $(74 \%)$, mialgias $(71 \%)$, odinofagia (48\%), deshidratación (5\%) e hipoxia (4\%).

Un 39\% de los pacientes tenía alguna co-morbilidad, $14 \%$ presentó co-morbilidad respiratoria, 9\% cardiovas- 
cular, 5\% hipotiroidismo y 5\% misceláneas. Algunos pacientes presentaban más de una patología como comorbilidad.

Del total, 13 pacientes estaban embarazadas al momento de la confirmación diagnóstica (10\%), con una edad promedio de 30,7 años, edad gestacional promedio de 28 semanas (rango: 7 a 38 semanas). Los síntomas más frecuentes previos a la consulta fueron fiebre, tos y mialgias. Todas las pacientes fueron hospitalizadas el mismo día de la consulta. No hubo complicaciones en este grupo y el monitoreo fetal durante la hospitalización resultó normal.

Un $90 \%$ de los pacientes fueron tratados con oseltamivir y en el caso de mujeres embarazadas, con zanamivir, en las primeras 48 horas de iniciados los síntomas.

Un $23 \%$ del total de pacientes, tenía antecedente de vacuna para influenza estacional 2009.

Al comparar los parámetros entre la población menor de 50 años $(n=95)$ y los de 50 años y más $(n=29)$, la mediana de edad fue de 30 y 64 años, respectivamente (Tabla 2).

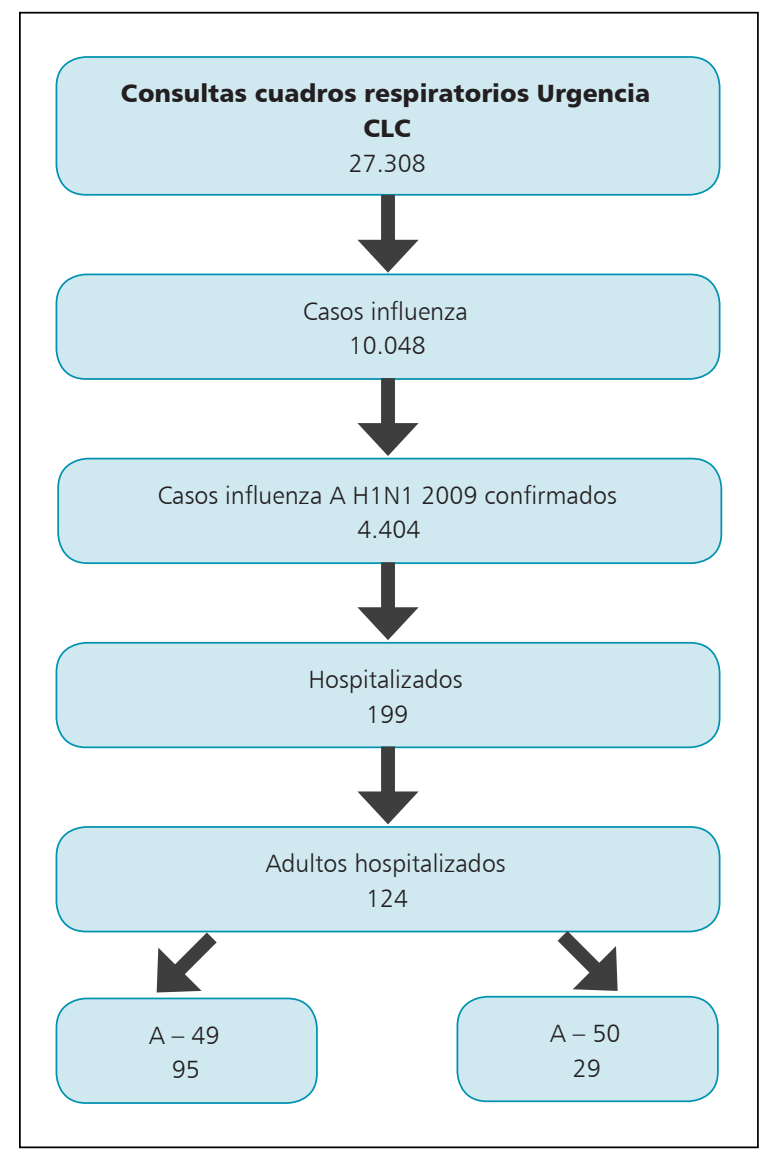

Figura 1. Casos de influenza A H1N1 2009 en adultos hospitalizados en Clínica Las Condes (17 mayo al 17 julio 2009).
Tabla 1. Características demográficas y clínicas de adultos hospitalizados por influenza A H1N1 2009 en Clínica Las Condes

\begin{tabular}{lc} 
Características & N $=\mathbf{1 2 4}$ \\
Sexo femenino $n^{\circ}(\%)$ & $80(64)$ \\
\hline Edad & 33 \\
$\quad$ Mediana - años & $15-82$ \\
Rango - años & \\
Grupo etáreo $n^{\circ}(\%)$ & $49(40)$ \\
$15-29$ años & $48(39)$ \\
$30-49$ años & $24(19)$ \\
$50-69$ años & 6 \\
$>70$ años &
\end{tabular}

Latencia en la consulta - días

Mediana

2

(p $25-75 \%)$

$1-4$

Signos y síntomas clínicos $n^{\circ}(\%)$

$\begin{array}{ll}\text { Fiebre } & 93(75)\end{array}$

$\begin{array}{ll}\text { Tos } & 92(74)\end{array}$

$\begin{array}{ll}\text { Mialgias } & 88(71)\end{array}$

Odinofagia $\quad 59(48)$

Deshidratación 6 (5)

Hipoxia $\quad 5$ (4)

Motivo de hospitalización $\mathrm{n}^{\circ}(\%)$

Compromiso de estado general $82(65)$

Respiratorio' 26 (21)

$\begin{array}{ll}\text { Digestivo }^{2} & 11(9)\end{array}$

Neurológico $^{3} \quad 2$ (2)

Cardiológico 1 (1)

Hematológico 1 (1)

Otorrinolaringológico 1 (1)

Co-morbilidad* $\mathrm{n}^{\circ}(\%) \quad 48$ (39)

$\begin{array}{ll}\text { Respiratorias }^{4} & 17(14)\end{array}$

Cardiovasculares $^{5} \quad 11$ (9)

Hipotiroidismo $\quad 6 \quad(5)$

Diabetes mellitus $\quad 3 \quad(2)$

Neurológicas $^{6} \quad 3 \quad 3(2)$

Inmunosupresión ${ }^{7} \quad 3$ (2)

Reumatológicas $^{8} \quad 2 \quad$ (2)

Hematológicas $^{9} \quad 1$ (1)

Nefrológicas $^{10} \quad 1$ (1)

Embarazo $n^{\circ}(\%) \quad 13(10)$

Vacunación anti-influenza $2009 \quad 29(23)$

Hospitalización - días

Mediana 3

(p $25-75 \%) \quad 2-3$

*Las condiciones descritas no son excluyentes. Algunos pacientes presentaban más de una patología simultáneamente. 'Incluye: asma descompensada, EPOC descompensada, neumonia, traqueítis y bronquitis obstructiva. ${ }^{2}$ Incluye dolor abdominal, colecistitis, sindrome diarreico agudo, hemorragia digestiva alta, hiperemesis, gastroenteritis aguda y gastroenteritis medicamentosa. ${ }^{3}$ ncluye lipotimia y sincope. ${ }^{4}$ Incluye asma (10), sindrome bronquial obstructivo (1), COP (1), EPOC (2), fibrosis pulmonar (1) y bronquiectasias (1). Incluye cardiopatía coronaria (2) e hipertensión arterial (9). IIncluye parálisis cerebral (1), meningioma operado (1) y retardo mental moderado (1). 7 Incluye trasplante bipulmonar (1), linfoma no Hodgkin (1) y mieloma múltiple (1). ${ }^{8}$ Corresponde a (1) lupus eritematoso sistémico y (1) artritis reumatoidea. ${ }^{9} \mathrm{Corresponde}$ a neutropenia febril. ${ }^{10} \mathrm{Corresponde}$ a pielonefritis aguda. 
Tabla 2. Caracterización demográfica y clínica de los grupos etáreos A-49 y A-50 hospitalizados por influenza A H1N1 2009 en Clínica Las Condes

\begin{tabular}{|c|c|c|}
\hline Características & $\begin{array}{c}<50 \text { años } \\
n=95\end{array}$ & $\begin{array}{c}\geq 50 \text { años } \\
n=29\end{array}$ \\
\hline Sexo femenino $n^{\circ}(\%)$ & $62(65)$ & $18(62)$ \\
\hline \multicolumn{3}{|l|}{ Edad } \\
\hline Mediana - años & 30 & 62 \\
\hline (p 25-75\%) - años & 9 & 9 \\
\hline \multicolumn{3}{|l|}{ Latencia en la consulta - días } \\
\hline Mediana & 2 & 2 \\
\hline$($ p 25-75\%) & $1-4$ & $2-4$ \\
\hline \multicolumn{3}{|l|}{ Signos y síntomas clínicos $n^{\circ}(\%)$} \\
\hline Mialgias & 71 (75) & $17(59)$ \\
\hline Fiebre & $71(75)$ & $22(76)$ \\
\hline Tos & $70(74)$ & $22(76)$ \\
\hline Odinofagia & $48(51)$ & $11(38)$ \\
\hline Deshidratación & $5(5)$ & $1(3)$ \\
\hline Hipoxia & $3(3)$ & $2(7)$ \\
\hline \multicolumn{3}{|l|}{ Motivo de hospitalización no (\%) } \\
\hline Compromiso de estado general & $66(69)$ & $16(55)$ \\
\hline Respiratorio ${ }^{1}$ & $17(18)$ & $9(31)$ \\
\hline Digestivo $^{2}$ & $10(11)$ & $1(3)$ \\
\hline Neurológico & $1(1)$ & $1(3)$ \\
\hline Cardiológico & - & $1(3)$ \\
\hline Hematológico & - & $1(3)$ \\
\hline Otorrinolaringológico & $1(1)$ & - \\
\hline \multicolumn{3}{|l|}{ Comorbilidad* $n^{\circ}(\%)$} \\
\hline Respiratorias ${ }^{3}$ & $9(9)$ & $8(28)$ \\
\hline Cardiovasculares ${ }^{4}$ & $1(1)$ & $10(34)$ \\
\hline Hipotiroidismo & $3(3)$ & $3(3)$ \\
\hline Diabetes mellitus & $2(2)$ & $4(14)$ \\
\hline Neurológicas ${ }^{5}$ & $2(2)$ & $1(3)$ \\
\hline Inmunosupresión & - & $3(10)$ \\
\hline Reumatológica ${ }^{6}$ & $1(1)$ & $1(3)$ \\
\hline Hematológica & $1(1)$ & - \\
\hline Nefrológica & $1(1)$ & - \\
\hline Embarazo $n^{\circ}(\%)$ & $13(14)$ & \\
\hline Vacunación anti-influenza 2009 & $23(24)$ & $6(21)$ \\
\hline \multicolumn{3}{|l|}{ Hospitalización-días } \\
\hline Mediana & 2 & 4 \\
\hline (p $25-75 \%)$ & $1.25-3$ & $2-4.7$ \\
\hline
\end{tabular}

*Las condiciones descritas no son excluyentes. Algunos pacientes presentaban más de una patología simultáneamente. ${ }^{1}$ A-49 Incluye: asma descompensada (3), EPOC descompensada (1), neumonía (11), traqueítis (1) y bronquitis obstructiva (1). A-50 Incluye: asma descompensada (1), EPOC descompensada (1), neumonía (5) y bronquitis obstructiva (2). ${ }^{2} \mathrm{~A}-49$ Incluye dolor abdominal (2), colecistitis (1), síndrome diarreico agudo (3), hiperemesis (2), gastroenteritis aguda (1) y gastroenteritis medicamentosa (1). A-50 Incluye hemorragia digestiva alta (1). ${ }^{3} \mathrm{~A}-49$ Incluye asma (7), síndrome bronquial obstructivo (1) y EPOC (1). A-50 Incluye asma (4), COP (1), EPOC (1), fibrosis pulmonar (1) y bronquiectasias (1). ${ }^{4} \mathrm{~A}-49$ Incluye hipertensión arterial (1). A-50 Incluye cardiopatía coronaria (2) e hipertensión arterial (8). ${ }^{5} \mathrm{~A}-49$ Incluye parálisis cerebral (1) y retardo mental moderado (1). A-50 Incluye meningioma operado (1). ${ }^{6} \mathrm{~A}-49$ Incluye lupus eritematoso sistémico (1). A-50 Incluye artritis reumatoidea (1).
La mediana de latencia para $<50$ fue de 2 días (p25 - 75\% = 1-4) y de 2 días (p25-75\%=2-4) para los $\geq 50$ años. La mediana de días de hospitalización fue significativamente mayor en el grupo $<50$ comparado con el grupo $\geq 50$ años ( 4 versus 2 días, respectivamente; $p<0,05$ ).

No hubo diferencias significativas en distribución por sexo, latencia, síntomas y signos ni en el motivo de hospitalización entre los dos grupos. El grupo $\geq 50$ presentó un mayor número de co-morbilidades, alcanzando una diferencia significativa al comparar ambos grupos $(\mathrm{p}<$ 0,05 ) (Tabla 2). Para $<50 \mathrm{y} \geq 50$ fue de tipo respiratorio en 9 y $28 \%$ respectivamente, cardiovascular 1 y $34 \%$, diabetes mellitus 2 y 14\%, inmunosupresión: sólo el grupo $\geq 50$ presentó $10 \%$; otras condiciones tuvieron poca relevancia.

En el grupo $<50$ sólo un paciente requirió de tratamiento en la Unidad de Cuidados Intensivos (UCI) con ventilación mecánica invasora y ECMO. En el grupo $\geq 50$ un solo paciente requirió sólo asistencia de ventilación mecánica invasora.

El antecedente de vacunación para influenza estacional 2009 fue similar para ambos grupos.

El principal motivo de hospitalización en ambos grupos fue $\mathrm{CEG}$, destacando mayor compromiso respiratorio en el grupo $\geq 50$ años.

\section{Discusión}

En el período comprendido entre el 17 de mayo y el 17 de julio del 2009, 124 pacientes adultos fueron hospitalizados en CLC por influenza A H1N1 2009. No se observaron grandes diferencias de género.

El principal motivo de hospitalización fue el CEG secundario a influenza y las principales co-morbilidades fueron afecciones respiratorias y cardiovasculares.

Al hacer el análisis por grupo etáreo, se observó un mayor número de casos hospitalizados en el grupo $<50$ y un mayor número de co-morbilidades en el grupo $\geq 50$, con menos casos hospitalizados. No hubo casos fatales. Es posible que esto último se deba a que los pacientes consultaron precozmente y a la disponibilidad de métodos de diagnóstico rápidos, unidos a la precocidad en el inicio del tratamiento antiviral.

La totalidad de los pacientes cursó una enfermedad respiratoria febril no complicada, de corta evolución, con síntomas y signos parecidos a la influenza estacional (cefalea, odinofagia, tos y mialgias), excepto dos casos con diagnóstico de neumonía grave en que fue necesario atención de UCI, con asistencia ventilatoria, y uno de ellos además con ECMO. Ambos evolucionaron de manera satisfactoria.

Un 9\% presentó compromiso digestivo como vómito y/o diarrea, incluso dolor abdominal, hechos que son 
muy poco frecuentes en la influenza estacional. El dolor abdominal, presente en nuestros pacientes, ha sido poco mencionado en la literatura médica ${ }^{4}$.

Con poca frecuencia se observaron síntomas como odontalgia, ardor traqueal, disestesias en muslos y artralgias, mas frecuentes en la influenza estacional de los últimos años. Varios pacientes sin antecedentes asmáticos refirieron sensación de pecho apretado y dolor torácico.

Se observó un mayor número de pacientes $<50$, con escasa co-morbilidad.

En el grupo $\geq 50$ hubo un menor número de pacientes. Es posible que esta menor incidencia se deba a que los pacientes mayores tengan un cierto grado de inmunidad por anticuerpos preexistentes ${ }^{6}$, especialmente quienes están por sobre los 60 años, nacidos antes de la pandemia de $1957^{4}$, o por inmunidad cruzada como sugieren los estudios serológicos con las vacunas estacionales de los últimos años ${ }^{7}$ Este grupo presentó una mayor comorbilidad y mayor número de neumonías.

Este diferente compromiso etáreo observado puede ser considerado al planificar las estrategias para enfrentar una eventual segunda ola del virus influenza A H1N1 2009.

No hemos encontrado en la literatura científica estudios que comparen este brote de influenza A H1N1 2009 entre menores y mayores de 50 años.

Una limitación en este estudio, al ser retrospectivo, fue que la información obtenida a partir de la base de datos, ficha clínica y notificaciones no siempre proporcionó toda la información requerida. La confirmación de los casos fue tanto por RPC como también mediante inmunocromatografía, porque hubo una alta correlación entre ambas según nuestro estudio en CLC, al correlacionar las primeras 196 muestras de prueba rápida versus RPC y que a su vez fueron confirmadas por el Instituto de Salud Pública.

Contar con una confirmación diagnóstica rápida, unido a la precocidad de consulta y la disponibilidad de tratamiento antiviral, pudo haber contribuído a que los pacientes cursaran una enfermedad de intensidad leve a moderada, con síntomas y signos parecidos a la influenza estacional, que en general tuvo un curso benigno con pocos días de hospitalización y sin casos fatales.

Agradecimientos. Los autores desean agradecer en forma especial a May Chomalí Garib, Directora de Servicios Médicos, por su importante colaboración en proporcionar información epidemiológica y acceso a la base de datos de Clínica Las Condes para la elaboración de este trabajo.

\section{Resumen}

Introducción: El nuevo virus influenza A H1N1 2009 ha mostrado un comportamiento clínico diferente en distintos grupos etáreos. Objetivo: Describir las características clínicas de los pacientes adultos hospitalizados con influenza A H1N1 2009 y comparar las variables clínicas y demográficas entre menores y mayores de 50 años. Pacientes y Método: Se incluyeron los pacientes hospitalizados entre el 17 de mayo y 17 de julio del 2009 confirmados por reacción de polimerasa en cadena (RPC) y/o pruebas rápidas para influenza A. Resultados: Se hospitalizaron 124 pacientes con una mediana de edad de 33 años (rango: 15-82 años). Un 36\% presentó co-morbilidad y los síntomas principales fueron: fiebre, tos, mialgias y odinofagia. Hubo 16 casos con neumonía. Del total, 77\% fueron $<50$ y $23 \% \geq 50$ años. Tenían co-morbilidades $21 \%$ en el grupo $<50$ versus $86 \%$ en $\geq 50$ años. No hubo casos fatales. Conclusiones: Los pacientes presentaron una enfermedad leve a moderada, de curso benigno y corta hospitalización. Hubo más hospitalizados en $<50$ y mayor co-morbilidad en $\geq 50$ años.

\section{Referencias}

1.- Organización Mundial de la Salud. Declaración de la Directora General de la OMS a la prensa [publicado 11 jun 2009]. Accedido el 14 agosto 2009. http://www.who.int/medicalcentre/news/ statements $/ 2009 / \mathrm{h} 1 \mathrm{n} 1_{\text {_ pandemic }}$ phase6 20090611/es/index.html

2.- Novel Swine-Origin Influenza A (H1N1) Virus Investigation Team. Emergence of a novel Swine-Origin Influenza A (H1N1) virus in humans. N Engl J Med. 2009 June 18; 360: 2605-15.

3.- Kilbourne E. Influenza Pandemics of the $20^{\text {th }}$ Century. Emerg Infect Dis. 2006; 12 (1): 9-14.

4.- Chowell G, Bertozzi S, Colchero MA, López-Gatell H, Alpuche-Aranda C,
Hernández M, Miller MA. Severe respiratory disease concurrent with the circulation of H1N1 influenza. N Eng J Med. 2009 Aug 13; 361;7: 674-9.

5.- Belshe R. Implications of the emergence of a novel H1 influenza virus. N Engl J Med 2009; June 18; 360: 2667-8.

6.- Centers for Disease Control and Prevention (CDC). Pandemic Influenza A (H1N1) Virus Infections-Chicago, Illinois, April-July 2009. MMWR Morb Mortal Wkly Rep 2009; 58 (33): 913-8.

7.- Hancock K, Veguilla V, Lu X. Zhong W, Butler EN, Sun H, et al. Cross-reactive antibody responses to the 2009 pandemic H1N1 influenza virus. N Eng J Med 2009; 361: 1-8.
8.- Torres JP, O`Ryan M. Herve B, Espinoza R, Acuña G, Mañalich J, Chomalí M. Impact of the novel influenza A (H1N1) during the 2009 autumn-winter season in a large hospital setting in Santiago, Chile. Clin Infect Dis 2010; 50 (6): 860-8.

9.- Gobierno de Chile. Ministerio de Salud. División de Prevención y Control de Enfermedades. Guía Clínica para el manejo de casos nueva influenza humana A (H1N1) (HA H1N1) etapa de mitigación. 9 Junio 2009.http://www.redsalud. gov.cl/portal/url/item/6b14a541236acdeae04001 011f010eda.pdf. (Accedido 30 septiembre 2009)

10.- Vasoo S, Stevens J, Singh K. Rapid antigen tests for diagnosis of pandemic (Swine) influenza A/H1N1. Clin Infect Dis 2009; 49 (7): 1090-3. 